\title{
Diabetes Mellitus and Its Associated Factors in Tuberculosis Patients in Maekel Region, Eritrea: Analytical Cross-Sectional Study
}

This article was published in the following Dove Press journal: Diabetes, Metabolic Syndrome and Obesity: Targets and Therapy

\author{
Zenawi Zeramariam Araia $\mathbb{D}^{1}$ \\ Araia Berhane Mesfin ${ }^{2}$ \\ Amanuel Hadgu Mebrahtu' \\ Adiam Ghebreyohanns \\ Tewelde $^{3}$ \\ Randa Osman ${ }^{4}$ \\ Hagos Andom Tuumzghi ${ }^{5}$ \\ 'National TB and Leprosy Control \\ Program, Communicable Disease \\ Control Division, Ministry of Health, \\ Asmara, Eritrea; ${ }^{2}$ Communicable \\ Diseases Control Division, Ministry of \\ Health, Asmara, Eritrea; ${ }^{3}$ Integrated \\ Disease Surveillance and Response, \\ Ministry of Health, Asmara, Eritrea; \\ ${ }^{4}$ Orrota College of Medicine and Health \\ Sciences, Asmara, Eritrea; ${ }^{5}$ Ministry of \\ Health, Maekel Region Branch, Asmara, \\ Eritrea
}

Background: Both diabetes mellitus (DM) and tuberculosis (TB) are among the leading causes of morbidity and mortality in Eritrea. TB-DM comorbidity is known to complicate TB care, control and prevention. However, systematically studied epidemiological data on TBDM comorbidity and its associated risk factors are lacking in this country.

Objective: This study aimed to assess the prevalence of DM and its associated factors among TB patients in the Maekel region, Eritrea.

Methods: Analytical cross-sectional study was conducted in eleven TB diagnostic and treatment sites. Pretested data extraction tool was used to collect data from medical records. Prevalence data were analysed using frequencies, proportions and median. To determine DM risk factors, univariable and multivariable logistic regression analysis was done with $95 \% \mathrm{CI}$ and $\mathrm{p}$ value $<0.05$ considered significant.

Results: Out of total eligible (1134) TB cases, DM prevalence was 9.88\%. Age and BMI were identified as independent risk factors for DM among TB patients. Higher odds of DM were found among TB patients aged 45-54 (aOR: 4.85[1.39-16.94], $\mathrm{p}=0.013$ ) and those $\geq 55$ (aOR: 6.99[2.12-23.04], $\mathrm{p}=0.001$ ). TB cases with normal BMI were two times more likely to have DM (aOR: $2.00[1.23-3.26], \mathrm{p}=0.005$ ) compared to those underweight.

Conclusion: The prevalence of DM among TB cases observed in this study is high, a clarion call to scale up current efforts to integrate TB-DM services within routine care. Furthermore, age and BMI were identified as independent risk factors for DM in TB cases, pointing to the need to pay attention to age and BMI status when managing this co-morbidity.

Keywords: TB-DM prevalence, transient hyperglycaemia, fasting blood glucose, prediabetes, Eritrea

\section{Background}

Tuberculosis (TB) is still a cause of public fear and stigma in many parts of the world accounting for millions of morbidity and mortality figures each year. ${ }^{1}$ The burden of this global epidemic is borne not by the developed countries where this disease is regarded as a disease of the past, but by low- and middle-income countries where ending TB is a distant reality. ${ }^{1-5}$ One of the key challenges in ending TB is the changing epidemiological and demographic transition with ageing populations, increasing burden of non-communicable diseases, exacerbating often overlooked comorbidities. $^{5,6}$

A preventable but incurable public health concern is the rise is Diabetes Mellitus (DM), with a global estimated 425 million cases in 2017 , predicted to
Correspondence: Zenawi Zeramariam Araia

National TB and Leprosy Control

Program, Communicable Diseases

Control Division, Ministry of Health,

Asmara, Eritrea

Tel +291-1-122129

Email zenhopncl@gmail.com
Diabetes, Metabolic Syndrome and Obesity: Targets and Therapy 2021:14 515-523

submit your manuscript

DovePres: in $\mathbf{P}$ 
increase to 629 million by 2045[3]. As a known risk factor, DM triples the risk of developing tuberculosis. $^{3,5-8}$ Consequently, $15 \%$ of the global TB cases are attributed to DM. ${ }^{9,10}$ In a recent systematic review on type $2 \mathrm{DM}$ and TB co-prevalence in low- and middle-income countries, the DM prevalence among TB patients ranged from $1.8 \%$ to $45 \%$, while TB prevalence among people with DM ranged from $0.1 \%$ to $6.0 \%,{ }^{11}$ underscoring the need for more focused attention to TBDM bidirectional screening programs in such settings. Nowadays, more TB patients are living with diabetes than concomitant HIV infection in the world. ${ }^{8}$ This increasing prevalence in TB-diabetes comorbidity offsets the expected gains of the intensified TB control efforts and presents a threat to the decades of progress made against TB. ${ }^{12}$ Therefore, the development of systematic approach to manage non-communicable diseases mainly diabetes mellitus in low-income countries is essential and timely. ${ }^{13}$

People with DM have a two- to three-fold risk of developing TB compared to those without DM. TB patients with DM have more severe clinical presentation and their treatment outcomes frequently worse than patients without DM, with higher risk of treatment failure and relapse. Additionally, DM may accelerate the emergence of drugresistant TB among those receiving TB treatment. ${ }^{3}$ Conversely, TB may increase existing insulin resistance, trigger the onset of diabetes in predisposed individuals and worsen glycaemic control in diabetic cases. Moreover, the effectiveness of TB and diabetes medications may be decreased due to drug-drug interactions, contributing to TB treatment failure and uncontrolled glycaemia. ${ }^{3}$ Thus, this double burden leads to substantial strain on individuals, families, society, and health systems. ${ }^{12}$

In Eritrea, the prevalence of DM in the general population is $3.8 \%{ }^{14}$ Moreover, the Health Management Information System report (HMIS) shows that DM was the leading cause of morbidity in Maekel region and ranked among the top five diseases in terms of mortality and morbidity at a national level in the year 2019. ${ }^{15} \mathrm{~TB}$ prevalence is as high as 123 per 100000 population. ${ }^{16} \mathrm{In}$ response to this dual epidemic, the country has adopted the World Health Organization (WHO) and International Union against TB and Lung Diseases (IULTD) collaborative frame-work for the care and control of TB and DM. ${ }^{9}$ One of the main components of this framework is bi-directional screening of TB and diabetes among patients. However, its implementation has not been as desired across the different regions of the country. There is dearth of literature in our setting on the burden of TBDM comorbidity and associated risk factors. Therefore, this study was conducted to assess the prevalence of DM among TB patients and its associated factors in Maekel region, to better inform more targeted interventions.

\section{Methods Study Design}

This study used analytical cross-sectional study design.

\section{Study Setting}

This study was conducted in Maekel, one of the six regions in Eritrea. It is densely populated and contains the state capital Asmara. The region was the first to carry out DM screening among TB patients and has relatively better quality of routine TB and DM data. The study subjects were all TB patients who were diagnosed with $\mathrm{TB}$ from 1st January 2016-31st December 2019 in all TB diagnostic and treatment sites of Maekel region (11 Health facilities in total). These health facilities included three community hospitals and eight health centres, most situated in Asmara. TB diagnosis was made based on the standard diagnostic procedures as defined in the National TB Control Program and WHO Guidelines. Thus, TB patients diagnosed, both bacteriologically confirmed (by smear microscopy and Xpert MTB/RIF) and clinically diagnosed (abnormalities by $\mathrm{X}$-ray, extra-pulmonary cases without laboratory confirmation and physicians' decision) were included in this study. ${ }^{17}$

As part of the integrate TB-DM services, TB patients are routinely screened for DM by collecting blood for fasting blood glucose test (overnight fasting) at the TB clinic. Those with fasting blood glucose level $\geq 126 \mathrm{mg} / \mathrm{dl}$ are referred to DM clinic for confirmation of DM diagnosis, follow-ups and appropriate management. On the other hand, DM patients are offered TB screening on every visits by the physician or clinician in the DM clinic. Consequently, presumptive TB are examined by the initial TB diagnostics (Acid Fast Bacilli Microscopy (AFB) and Xpert MTB/RIF concurrently including others as appropriate). Those who were found to have TB are linked to the TB services and treated according to the standard TB management. At the same time, all TB presumptive are offered HIV testing and counselling services at the TB clinic.

\section{Study Population}

All TB cases who were diagnosed and registered in the national tuberculosis treatment register during the period 
of 1st January 2016-31st December 2019 were included in the study. However, paediatric TB cases $(<15$ years old) and those with unknown or undocumented diabetic status were excluded from the final analysis.

A total of 1349 TB patients were diagnosed and treated for TB from 1st January 2016 to 31st December 2019 in all the TB diagnostic and treatment sites in Maekel region. Of these, TB cases who met the inclusion criteria were included in the study. Thus, the final sample size of the study was 1134 after excluding 215 TB patients with unknown or undocumented status of diabetes mellitus and paediatric TB patients.

\section{Variables, Instruments and Data Collection}

In this study, DM was the outcome variable and the sociodemographic and clinical variables were the risk factors for DM among TB patients. A pre-tested structured data abstraction tool was used to collect the following clinical and socio-demographic variables from registers (TB treatment register, TB laboratory register, TB treatment card) and surveillance reports (quarterly TB patients' nutritional assessment and diabetes report, and quarterly TB notification and outcome report): age, sex, residence, height (in meter), weight (in kilogram), type of TB patient, TB site, TB treatment group, body mass index, fasting blood sugar (FBS) test results, HIV status, ART and CPT, and sputum smear results.

BMI results were classified as underweight $(<18.5)$, normal weight (18.5-24.9), overweight (25-29.9) and obese $(>30) .{ }^{18}$ The DM screening result which was collected from the TB patients' medical record (TB treatment card and quarterly TB patients' nutritional assessment and diabetes report) was classified using the WHO diagnostic criteria for DM. Hence, fasting blood glucose level $\geq$ $126 \mathrm{mg} / \mathrm{dl}$ was considered as DM and fasting blood glucose levels of $110-125 \mathrm{mg} / \mathrm{dl}$ as pre-diabetes. ${ }^{19}$

\section{Data Analysis}

Anonymised data were entered and analysed using IBM SPSS Statistics for Windows, version 23 (IBM Corp., Armonk, N.Y., USA). A logic check was used to identify errors made during data entry, and appropriate corrections made. Categorical data were analysed using frequencies

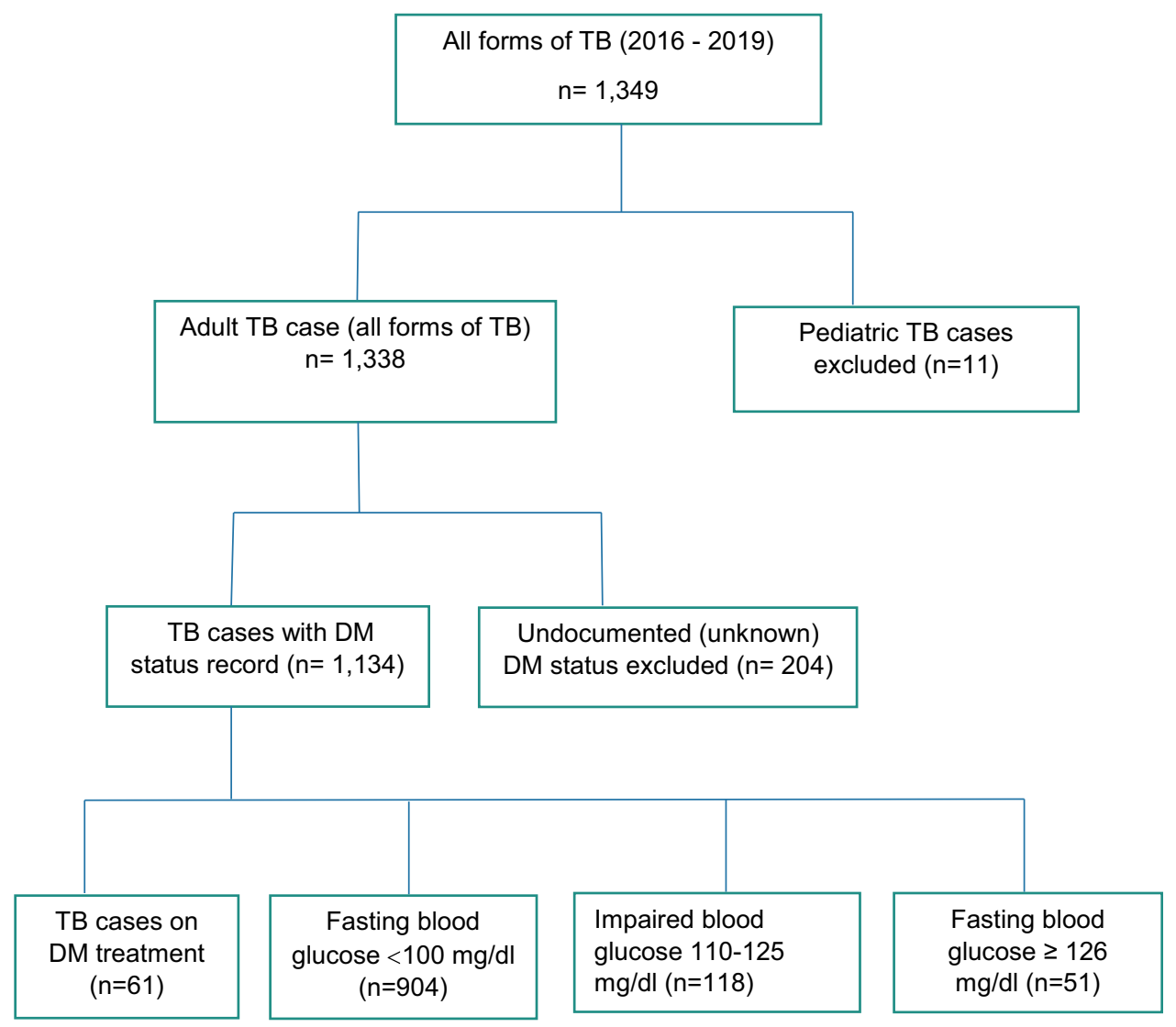

Figure I Flow chart on step by step selection and final analysis of study participants. 
and proportions, and Chi-square test used to assess for any association of DM with socio-demographic and clinical characteristics. To determine the strength of association, both univariable and multivariable logistic regression analysis was performed, with significance level set at 5\%.

\section{Results}

Between 2016 and 2019, there were 1349 registered TB patients at the study sites. A total of 204 (15.12\%) had no documented blood glucose test results and an additional 11 were children $<15$ years and thus excluded in the final analysis. A total of 1134 were eligible for the study and included in the final analysis (Figure 1).

\section{Demographic Profile of Study Participants}

Among the study participants, 608 (53.6\%) were males. The median age was 43 years (interquartile range 30-60), with a third (342) aged $\geq 55$ years. Close to two-thirds (597) had BMI $<18.5$ and 140 (12.4\%) were HIV positive. The majority $(72.5 \%)$ resided in urban areas. Almost all (93.2\%) were new TB patients and 751 (66.3\%) were diagnosed with pulmonary TB (Table 1).

\section{Prevalence of DM Among TB Cases}

The prevalence of DM among TB patients was 112/1134 (9.9\%). Among those with both TB and DM, 61 (54.5\%) were known DM and the rest diagnosed during routine DM screening. In addition, 118 (10.4\%) TB cases were found to be in the pre-diabetes stage (Figure 1).

DM prevalence was higher among males, 70 (11.5\%) compared to females, $42(8.0 \%)$ and increased with age from $2 \%$ in the $15-24$ age group to $17.3 \%$ among those $\geq 55$ years. Prevalence also increased with BMI, from 5.9\% among those underweight to $25 \%$ among those obese. There was no difference in point prevalence by place of residence, type of TB or HIV status (Table 2).

\section{Factors Associated with DM Among TB Cases}

The factors associated with being DM are summarized in Table 3. Compared to the younger 15-24 age group, being older, 45-54 and $\geq 55$ was associated with 4.9 and 7 -fold odds (aOR: 4.9[1.4-16.9], $\mathrm{p}=0.013$ ) and (aOR: 7.0[2.1-23.0], $\mathrm{p}=$ 0.001) of being DM, respectively. Compared to those underweight, having a normal BMI was associated with 2-fold odds (aOR: 2.00[1.23-3.26], $\mathrm{p}=0.005$ ) of being DM.
Table I Socio-Demographic and Clinical Characteristics of TB Patients Enrolled in the Study in Maekel Region, 2016-2019

\begin{tabular}{|c|c|c|}
\hline Variables & Frequency & $\begin{array}{l}\text { Percentage } \\
\text { (\%) }\end{array}$ \\
\hline \multicolumn{3}{|l|}{ Sex } \\
\hline Male & 608 & $53.6 \%$ \\
\hline Female & 526 & $46.4 \%$ \\
\hline \multicolumn{3}{|l|}{ Age range } \\
\hline $15-24$ & 150 & $13.2 \%$ \\
\hline $25-34$ & 234 & $20.6 \%$ \\
\hline $35-44$ & 225 & $19.8 \%$ \\
\hline $45-54$ & 183 & $16.1 \%$ \\
\hline$\geq 55$ & 342 & $30.2 \%$ \\
\hline \multicolumn{3}{|l|}{ Residence } \\
\hline Urban & 822 & $72.5 \%$ \\
\hline Rural & 312 & $27.5 \%$ \\
\hline \multicolumn{3}{|l|}{$\begin{array}{l}\text { Body mass index (BMI) } \\
(n=999)\end{array}$} \\
\hline Underweight $(<18.5)$ & 597 & $59.8 \%$ \\
\hline Normal (18.5-24.9) & 347 & $34.7 \%$ \\
\hline Overweight (25-29.9) & 51 & $5.1 \%$ \\
\hline Obese $(\geq 30)$ & 4 & $0.4 \%$ \\
\hline \multicolumn{3}{|l|}{ Type of TB patient $(n=1 \mid 33)$} \\
\hline New & 1056 & $93.2 \%$ \\
\hline Previously treated & 77 & $6.8 \%$ \\
\hline \multicolumn{3}{|l|}{ TB sites $(n=1 \mid 33)$} \\
\hline Pulmonary TB & 751 & $66.3 \%$ \\
\hline Extra-pulmonary TB & 382 & $33.7 \%$ \\
\hline \multicolumn{3}{|l|}{$\begin{array}{l}\text { TB treatment category } \\
(n=|| 33)\end{array}$} \\
\hline Category-I & 1056 & $93.2 \%$ \\
\hline Category-II & 77 & $6.8 \%$ \\
\hline \multicolumn{3}{|l|}{ HIV test result $(n=1 \mid 32)$} \\
\hline Negative & 992 & $87.6 \%$ \\
\hline Positive & 140 & $12.4 \%$ \\
\hline
\end{tabular}

\section{Discussion}

In the absence of systematically studied information on TB-DM comorbidity in our setting, we conducted a study with the aim of assessing the magnitude of diabetes mellitus and its associated risk factors in TB patients. This study has provided valuable information on the burden and related risk factor of DM in TB patients, which emphasizes a need for the implementation of age and BMI focused intervention. 
Table 2 Comparison Among TB-DM and TB without DM Cases in Maekel Region, 2016-2019

\begin{tabular}{|c|c|c|c|}
\hline \multicolumn{3}{|l|}{ TB Patients $(n=1 \mid 34)$} & \multirow[t]{2}{*}{ p-value $(\chi 2$ Test) } \\
\hline Variables & Diabetic $(n=|| 2)$ & Non-Diabetic $(n=1022)$ & \\
\hline \multicolumn{4}{|l|}{ Sex } \\
\hline Male & $70(11.5 \%)$ & $538(88.5 \%)$ & 0.047 \\
\hline Female & $42(8.0 \%)$ & $484(92.0 \%)$ & \\
\hline \multicolumn{4}{|l|}{ Age range } \\
\hline $15-24$ & $3(2.0 \%)$ & $147(98.0 \%)$ & $<0.001$ \\
\hline $25-34$ & $8(3.4 \%)$ & $226(96.6 \%)$ & \\
\hline $35-44$ & $19(8.4 \%)$ & $206(91.6 \%)$ & \\
\hline $45-54$ & $23(12.6 \%)$ & $160(87.4 \%)$ & \\
\hline$\geq 55$ & $59(17.3 \%)$ & $283(82.8 \%)$ & \\
\hline \multicolumn{4}{|c|}{ Body mass index (BMI) $(n=999)$} \\
\hline Underweight $(<18.5)$ & $35(5.9 \%)$ & $562(94.1 \%)$ & 0.005 \\
\hline Normal(I8.5-24.9) & $40(11.5 \%)$ & $307(83.5 \%)$ & \\
\hline Overweight(25-29.9) & $7(13.7 \%)$ & $44(86.3 \%)$ & \\
\hline Obese $(\geq 30)$ & $\mathrm{I}(25.0 \%)$ & $3(75.0 \%)$ & \\
\hline \multicolumn{4}{|l|}{ Residence } \\
\hline Urban & $84(10.2 \%)$ & $738(89.8 \%)$ & 0.530 \\
\hline Rural & $28(9.0 \%)$ & $284(91.0 \%)$ & \\
\hline \multicolumn{4}{|c|}{ Type of TB patient $(n=1 \mid 33)$} \\
\hline New & $103(9.8 \%)$ & $953(90.3 \%)$ & 0.583 \\
\hline Previously treated & $9(\mid 1.7 \%)$ & $68(88.3 \%)$ & \\
\hline \multicolumn{4}{|l|}{ TB sites $(n=1 / 33)$} \\
\hline Pulmonary TB & $83(11.1 \%)$ & 668(88.9\%) & 0.065 \\
\hline Extra-pulmonary TB & $29(7.6 \%)$ & $353(92.4 \%)$ & \\
\hline \multicolumn{4}{|c|}{ TB treatment category $(n=|| 33)$} \\
\hline Category-I & $103(9.8 \%)$ & $953(90.2 \%)$ & 0.583 \\
\hline Category-II & $9(\mid 1.7 \%)$ & $68(88.3 \%)$ & \\
\hline \multicolumn{4}{|c|}{ HIV test result $(n=|| 32)$} \\
\hline Negative & $101(10.2 \%)$ & $891(89.8 \%)$ & 0.389 \\
\hline Positive & $\mathrm{II}(7.9 \%)$ & $129(92.1 \%)$ & \\
\hline
\end{tabular}

The prevalence of DM among TB cases in the current study was $9.9 \%$ which is more than two folds higher than the occurrence of DM in the general population of Eritrea. ${ }^{14}$ This is consistent to findings from Tanzania (9.7\%) and pooled DM prevalence of Sub-Sahara Africa $(9 \%)^{20,21}$ but lower than the pooled global DM prevalence $(15.3 \%)$ in patients with active tuberculosis. ${ }^{22}$ Contradicting results of lower ${ }^{23-26}$ and higher DM prevalence among TB cases were also reported in different countries. $^{27-29}$ The difference in TB-DM comorbidity in these studies could be due to the inherent difference in socio-economic and demographic characteristics of the studied population and the existing burden of both diseases in their respective countries. Likewise, variation in diagnostic methods for DM among the different studies could have affected the reported prevalence.

In this study, $51(45.55 \%)$ of the TB-DM cases were not aware of their DM condition before TB diagnosis. This is much higher than earlier finding from Ethiopia (6.4\%) but lower than the reports from Tanzania (60\%) and Kenya $(69.5 \%)^{20,30,31}$ Despite the fact that our study did not provide data on the types of DM among TB cases, most DM patients in Eritrea are suffering from type 2 DM. ${ }^{32}$ Due to its gradual development, most patients with Type 2 DM experience minimal symptoms. ${ }^{33}$ This could in turn contribute to delayed healthcare seeking among patients, making Type 2 DM often undiagnosed in TB cases. ${ }^{34,35}$ This routine DM screening in TB patients aided to find 
Table 3 Univariable and Multivariable Logistic Regression of Risk Factors for DM Among TB Cases in Maekel Region, 2016-2019

\begin{tabular}{|c|c|c|c|c|}
\hline \multirow[t]{2}{*}{ Variables } & \multicolumn{3}{|c|}{ TB Patients $(n=\mid 134)$} & \multirow[b]{2}{*}{$\operatorname{aOR}[95 \% \mathrm{Cl}]$} \\
\hline & $D M(n=|| 2)$ & Non-DM $(n=1022)$ & $\operatorname{cOR}[95 \% \mathrm{CI}]$ & \\
\hline \multicolumn{5}{|l|}{ Sex } \\
\hline Male & $70(11.5 \%)$ & $538(88.5 \%)$ & $1.50[1.00-2.24]^{*}$ & $1.27[0.79-2.04]$ \\
\hline Female & $42(8.0 \%)$ & $484(92.0 \%)$ & I & I \\
\hline \multicolumn{5}{|l|}{ Age range } \\
\hline I5-24 & $3(2.0 \%)$ & $147(98.0 \%)$ & I & 1 \\
\hline $25-34$ & $8(3.4 \%)$ & $226(96.6 \%)$ & $1.74[0.45-6.64]$ & I.19[0.29-4.87] \\
\hline $35-44$ & $19(8.4 \%)$ & $206(91.6 \%)$ & $4.52[1.31-15.55]^{*}$ & $2.66[0.73-9.68]$ \\
\hline $45-54$ & $23(12.6 \%)$ & $160(87.4 \%)$ & $7.04[2.07-23.95]^{* *}$ & $4.85[1.39-16.94]^{*}$ \\
\hline$\geq 55$ & $59(17.3 \%)$ & $283(82.8 \%)$ & $10.22[3.15-33.15]^{* * *}$ & $6.99[2.12-23.04]^{* *}$ \\
\hline \multicolumn{5}{|c|}{ Body mass index (BMI) } \\
\hline$<18.5$ & $35(5.9 \%)$ & $562(94.1 \%)$ & 1 & 1 \\
\hline $18.5-24.9$ & $40(11.5 \%)$ & $307(83.5 \%)$ & $2.09[1.30-3.36]^{* *}$ & $2.00[1.23-3.26]^{* *}$ \\
\hline $25-29.9$ & $7(13.7 \%)$ & $44(86.3 \%)$ & $2.56[1.07-6.08]^{*}$ & $2.13[0.88-5.17]$ \\
\hline$\geq 30$ & $\mathrm{I}(25.0 \%)$ & $3(75.0 \%)$ & $5.35[0.54-52.79]$ & $7.62[0.69-84.01]$ \\
\hline \multicolumn{5}{|c|}{ HIV test result } \\
\hline Negative & $101(10.2 \%)$ & $891(89.8 \%)$ & $1.33[0.69-2.54]$ & \\
\hline Positive & $\mathrm{II}(7.9 \%)$ & $129(92.1 \%)$ & I & \\
\hline
\end{tabular}

Notes: $* \mathrm{P}<0.05 ; * * \mathrm{P}<0.01 ; * * * \mathrm{P}<0.001$.

Abbreviations: cOR, crude odds ratio; aOR, adjusted odds ratio; $\mathrm{Cl}$, confidence interval; $\mathrm{BMI}$, body mass index.

DM cases, who may have otherwise been missed or presented later with complicated DM.

The impaired fasting blood glucose (pre-diabetes) identified in one-tenth of the TB cases supports previous study which found comparable ${ }^{36}$ result but opposes studies which reported lower ${ }^{37,38}$ and higher rates elsewhere. ${ }^{30,31,39-41}$ Studies indicate that temporary hyperglycaemia in TB cases could occur as a result of a stress reaction to TB infection and hyperglycaemic effect of some anti-TB drugs. ${ }^{35,42,43}$ Furthermore, impaired glucose tolerance or new DM could result from TB pancreatitis and TB-related endocrine hypofunction. ${ }^{35}$ But this hyperglycaemic condition could partly or completely returned to a normal glucose level after TB treatment completion. ${ }^{35,42,43}$ Hence, the DM and pre-diabetes prevalence in this study could have been overestimated as the new DM and pre-diabetes cases were not screened for DM after TB treatment completion.

In this study, age and BMI were independently associated with DM among TB cases. TB cases aged 45-54 and $\geq 55$ were 5 and 7 times more likely to have DM, respectively, in comparison to younger TB cases. This finding is consistent to previous studies which noted higher odds of DM in older TB patients. ${ }^{20,25,39}$ On the other hand, a study conducted in Ethiopia found that not only old but young TB patients aged 26-40 had higher probabilities of being diabetic. ${ }^{30}$ Nearly three fourth of the TB-DM cases in the current study were $\geq 45$ years old but this age group account for only $46 \%$ of the study population. This can be explained by the fact that Type 2 DM mostly affects older adults and also increasing age is a risk factor for both TB and $\mathrm{DM}^{30}$ In addition, a decline in immune function as a result of ageing ${ }^{44-46}$ could have increased their vulnerability to develop both diseases.

TB patients with normal weight were two times more likely to have DM as compared to underweight TB cases. This is equivalent to prior findings which stated normal weight TB patients had higher odds of DM than underweight TB cases. ${ }^{29,40}$ In contrast, increased odds of DM among severe underweight male $\mathrm{TB}$ cases, ${ }^{47}$ nonsignificant BMI difference ${ }^{31,48}$ and significant higher $\mathrm{BMI}^{49,50}$ were also documented. Even though the number of overweight and obese TB cases in this study was almost negligible, which partly could have been affected by the possible weight loss related to TB induced hyperglycaemia, it is important to note that excessive weight gain is recognized as a predisposing factor to diabetes and it is an independent risk factor for TB. ${ }^{3,49}$ It is also suggested that some people with normal body composition may have been disposed to diabetes either through unfortunate 
metabolic programming from a history of malnutrition which is common in Sub-Sahara Africa ${ }^{51}$ or from being hereditarily susceptible to DM at lower BMI. ${ }^{47}$

\section{Conclusions}

This study showed a significant prevalence of DM in TB cases in Maekel region of Eritrea. Almost half of the DM cases were identified by the routine DM screening in TB patients. This indicates a need for the provision of integrated service, a key and timely intervention for tackling this dual burden. Age and BMI were determinants of DM in TB patients. Full-scale implementation of the already initiated DM screening in TB cases should therefore be strengthened with due focus on age and BMI status and more sensitive testing methods should be used in all health facilities.

\section{Limitations of the Study}

Due to the nature of the study, data were collected retrospectively from existing TB registers and medical records. Thus, some information may be missing. The use of FBG for the diagnosis of DM which is less accurate than HbA1C could have overestimated the prevalence of DM in this study. This study finding is only generalizable to Maekel region and as children were not included it cannot be interpolated to such population.

\section{Abbreviations}

AFB, acid fast bacilli; aOR, adjusted odds ratio; BMI, body mass index; $\mathrm{CI}$, confidence interval; cOR, crude odds ratio; DM, diabetes mellitus; FBG, fasting blood glucose; FBS, fasting blood sugar; HbAlc, glycosylated haemoglobin; HIV, human immune deficiency virus; HMIS, health management information system; IDF, international diabetes federation; IUATLD, international union against $\mathrm{TB}$ and lung disease; IQR, interquartile ranges; $\mathrm{MOH}$, ministry of health; SPSS, statistical package for social sciences; TB, tuberculosis; WHO, World Health Organization.

\section{Data Sharing Statement}

The data set analysed in this study is available from the corresponding author on reasonable request.

\section{Ethics Approval and Consent to Participate}

The study was conducted in accordance with the Declaration of Helsinki. Ethical approval was obtained from the Health Research and Ethical committee of the Ministry of Health, Eritrea. Study participants' informed consent was waived by the ethical committee due to the retrospective nature of the data review. The data was collected anonymously and did not include any personal identifiers to ensure confidentiality.

\section{Acknowledgments}

The authors would like to thank all TB focal persons in Maekel Region, Soliaya Kidane, Nahom Kiros and Meron Mehari for their support.

\section{Author Contributions}

ZZA conceived the research question, study design, performed data analysis and interpretation, and wrote drafted the manuscript. All authors made a substantial contribution to the work reported, that is in the study design, execution, acquisition of data, interpretation, took part in drafting, revising and critically reviewing the article; gave final approval of the version to be published; have agreed on the journal to which the article has been submitted; and agree to be accountable for all aspects of the work.

\section{Disclosure}

The authors declare that there is no conflict of interest regarding the publication of this paper.

\section{References}

1. World Health Organization. Global Tuberculosis Report. Licence: CC BY-NC-SA 3.0 IGO. 2018.

2. Lee SH. Tuberculosis infection and latent tuberculosis. Tuberc Respir Dis. 2016;79(4):201-206. doi:10.4046/trd.2016.79.4.201

3. Lin Y, Harries AD, Kumar AMV, et al. Management of Diabetes Mellitus-Tuberculosis: A Guide to the Essential Practice. 1st ed. Paris, France: IUATLD; 2019.

4. World Health Organization. Treatment of Tuberculosis Guidelines. WHO/HTM/TB/2009. 4th ed. Vol. 420. 2009.

5. Harries AD, Satyanarayana S, Kumar AMV, et al. Epidemiology and interaction of diabetes mellitus and tuberculosis and challenges for care: a review. Public Health Action. 2013;3(1):3-9. doi:10.5588/ pha.13.0024

6. Restrepo BI. Diabetes and tuberculosis. Microbiol Spectr. 2016;4(6). doi:10.1128/microbiolspec.TNMI7-0023-2016

7. Dooley KE, Chaisson RE. Tuberculosis and diabetes mellitus: convergence of two epidemics. Lancet Infect Dis. 2009;9(737-46.11):737-746. doi:10.1016/S1473-3099(09)70282-8

8. Girardi E, Shepisi MS, Goletti D, et al. The global dynamics of diabetes and tuberculosis: the impact of migration and policy implication. Int $J$ Infect Dis. 2017;56:45-53. doi:10.1016/j. ijid.2017.01.018

9. World Health Organization and International Union against Tuberculosis and Lung Diseases. Collaborative Framework for Care and Control of Tuberculosis and Diabetes. WHO/HTM/TB/2011.15. 2011. 
10. World Health Organization. The dual epidemic of TB and diabetes; 2016. Available from: https://www.who.int/tb/publications/diabetes_ tb.pdf. Accesed July 6, 2019.

11. McMurry HS, Mendenhall E, Rajendrakumar A, Nambiar L, Satyanarayana S, Shivashankar R. Co-prevalence of type 2 diabetes mellitus and tuberculosis in low-income and middle-income countries: a systematic review. Diabetes Metab Res Rev. 2019;35(1): e3066. doi:10.1002/dmrr.3066

12. Lönnroth K, Roglic G, Harries AD. Improving tuberculosis prevention and care through addressing the global diabetes epidemic: from evidence to policy and practice. Lancet Diabetes Endocrinol. 2014;2 (9):730-739. doi:10.1016/S2213-8587(14)70109-3

13. Gennaro FD, Marotta C, Antunes M, Pizzol D. Diabetes in active tuberculosis in low-income countries: to test or to take care? Lancet Glob Health. 2019;7(6):e707. doi:10.1016/S2214-109X(19)30173-1

14. World Health Organization. Diabetes country profiles; 2016. Available from: http://origin.who.int/diabetes/country-profiles/eri_en. pdf. Accessed August 7, 2019.

15. MOH. Annual Health Service Activity Report. Asmara, Eritrea: Health Management Information System (HMIS). NHIS division; 2019.

16. World Health Organization. Global Tuberculosis Report. Country Profile. WHO/HTM/TB/2071.23. Geneva, Switzerland; 2017.

17. World Health Organization. Definitions and Reporting Framework for Tuberculosis-2013 Revision. WHO/HTM/TB/2013.2. 2013.

18. WHO. Report of a World Health Organization Expert Committee. Physical Status: The Use and Interpretation of Anthropometry. Technical Report Series No. 854. 1995.

19. WHO. Definition and Diagnosis of Diabetes Mellitus and Intermediate Hyperglycemia. Report of a WHO/IDF Consultation. Geneva, Switzerland; 2006.

20. Munseri PJ, Kimambo H, Pallangyo K. Diabetes mellitus among patients attending TB clinics in Dar es Salaam: a descriptive cross-sectional study. BMC Infect Dis. 2019;19(1):915. doi:10.1186/ s12879-019-4539-5

21. Alebel A, Wondemagegn AT, Tesema C, et al. Prevalence of diabetes mellitus among tuberculosis patients in Sub-Saharan Africa: a systematic review and meta-analysis of observational studies. BMC Infect Dis. 2019;19(1):254. doi:10.1186/s12879-019-3892-8

22. Noubiap JJ, Nansseu JR, Nyaga UF, et al. Global prevalence of diabetes in active tuberculosis: a systematic review and metaanalysis of data from $2 \cdot 3$ million patients with tuberculosis. Lancet Glob Health. 2019;7(4):e448-60. doi:10.1016/S2214-109X(18) 30487-X

23. Fwoloshi S, Hachaambwa LM, Chiyeñu KO, et al. Screening for diabetes mellitus among tuberculosis patients: findings from a study at a tertiary hospital in Lusaka, Zambia. Can J Infect Dis Med Microbiol. 2018;2018:1-5. doi:10.1155/2018/3524926

24. Getachew A, Mekonnen S, Alemu S. High magnitude of diabetes mellitus among active pulmonary tuberculosis patients in Ethiopia. Br J Med Med. 2014;4(3):862-872.

25. Akkerman OW, de Lange WCM, Schölvinck EH. Prevalence and factors associated with diabetes mellitus among tuberculosis patients: a nationwide cohort. Eur Respir J. 2016;48(1):261-264. doi:10.1183/ 13993003.00112-2016

26. Pizzol D, Gennaro FD, Chhaganlal KD, et al. Prevalence of diabetes mellitus in newly diagnosed pulmonary tuberculosis in Beira, Mozambique. Afr Health Sci. 2017;17(3):773-779. doi:10.4314/ahs.v17i3.20

27. Jiménez-Corona ME, Cruz-Hervert LP, García-García L. Association of diabetes and tuberculosis: impact on treatment and post-treatment outcomes. Thorax. 2013;68(3):214-220. doi:10.1136/thoraxjnl-2012202976

28. Alavi SM, Khoshkhoy MM. Pulmonary tuberculosis and diabetes mellitus: co-existence of both diseases in patients admitted in a teaching hospital in the southwest of Iran. Caspian J Intern Med. 2012;3(2):421-424.
29. Pande T, Huddart S. Prevalence of diabetes mellitus amongst hospitalized tuberculosis patients at an Indian tertiary care center: a descriptive analysis. PLoS One. 2018;13(7):e0200838. doi:10.1371/journal.pone. 0200838

30. Tenaye L, Mengiste B, Baraki N, Mulu E. Diabetes mellitus among adult tuberculosis patients attending tuberculosis clinics in eastern Ethiopia. Biomed Res Int. 2019;2019:1-7. doi:10.1155/2019/7640836

31. Owiti P, Keter A, Harries AD, et al. Diabetes and pre-diabetes in tuberculosis patients in western Kenya using point-of-care glycated haemoglobin. Public Health Action. 2017;7(2):147-154. doi:10.5588/ pha.16.0114

32. Seyum B, Mebrahtu G, Usman A, et al. Profile of patients with diabetes in Eritrea: results of first phase registry analyses. Acta Diabetol. 2010;47(1):23-27. doi:10.1007/s00592-009-0093-8

33. World Health Organization. Classification of Diabetes Mellitus. Licence: CC BY-NC-SA 3.0 IGO. Geneva; 2019.

34. Ruslami R, Aarnoutse RE, Alisjahbana B, Van Der Ven AJAM, Van Crevel R. Implications of the global increase of diabetes for tuberculosis control and patient care. Trop Med Int Health. 2010;15 (11):1289-1299. doi:10.1111/j.1365-3156.2010.02625.x

35. Baghaei P, Marjani M, Javanmard P, Tabarsi P, Masjedi MR. Diabetes mellitus and tuberculosis facts and controversies. J Diabetes Metab Disord. 2013;12(58). doi:10.1186/2251-6581-12-58

36. Workneh MH, Bjune GA, Yimer SA, Pai M. Prevalence and associated factors of diabetes mellitus among tuberculosis patients in southeastern Amhara region, Ethiopia: a cross sectional study. PLoS One. 2016;11(1): 0147621.

37. Li L, Lin Y, Mi F, et al. Screening of patients with tuberculosis for diabetes mellitus in China. Trop Med Int Health. 2012;17 (10):1294-1301. doi:10.1111/j.1365-3156.2012.03068.x

38. Achanta S, Tekumalla RR, Jaju J, et al. Screening tuberculosis patients for diabetes in a tribal area in South India. Public Health Action. 2013;3(suppl 1):43-47. doi:10.5588/pha.13.0033

39. Hoa NB, Phuc PD, Hien NT, et al. Prevalence and associated factors of diabetes mellitus among tuberculosis patients in Hanoi, Vietnam Hanoi, Vietnam. BMC Infect Dis. 2018;18(603). doi:10.1186/s12879018-3519-5

40. Viswanathan V, Kumpatla S, Aravindalochanan V, et al. Prevalence of diabetes and pre-diabetes and associated risk factors among tuberculosis patients in India. PLoS One. 2012;7(7):e41367. doi:10.1371/ journal.pone. 0041367

41. Oni T, Berkowitz N, Kubjane M, Rene Goliath NS, Levitt RJ, Wilkinson RJ. Trilateral overlap of tuberculosis, diabetes and HIV-1 in a high-burden African setting: implications for TB control. Eur Respir J. 2017;50(1):1700004. doi:10.1183/13993003.00004-2017

42. Yorke E, Atiase Y, Akpalu J, Sarfo-Kantanka O, Boima V, Dey ID. The bidirectional relationship between tuberculosis and diabetes. Tuberc Res Treat. 2017;2017. doi:10.1155/2017/1702578.

43. Noémie Boillat-Blanco KL, Ramaiya MM, Mganga M, et al. Transient hyperglycemia in patients with tuberculosis in tanzania: implications for diabetes screening algorithms. JID. 2016;213 (7):1163-1172. doi:10.1093/infdis/jiv568

44. Negin J, Abimbola S, Marais BJ. Tuberculosis among older adultstime to take notice. Int J Infect Dis. 2015;32:135-137. doi:10.1016/j. ijid.2014.11.018

45. Yoshikawa Thomas T, Rajagopalan S. Tuberculosis and aging: a global health problem. Clin Infect Dis. 2001;33(7):1034-1039. doi: $10.1086 / 322671$

46. Montecino-Rodriguez E, Berent-Maoz B, Dorshkind K. Causes, consequences, and reversal of immune system aging. $J$ Clin Invest. 2013;123(3):958-965. doi:10.1172/JCI64096

47. Faurholt-Jepsen D, Range N, PrayGod G, et al. The role of anthropometric and other predictors for diabetes among urban Tanzanians with tuberculosis. Int J Tuberc Lung Dis. 2012;16(12):1680-1685. doi:10.5588/ijtld.12.0360 
48. Agarwal AK, Gupta G, Marskole P, Agarwal A. A study of the patients suffering from tuberculosis and tuberculosis-diabetes comorbidity in revised national tuberculosis control program centers of northern Madhya Pradesh, India. Indian J Endocr Metab. 2017;21 (570-6):570. doi:10.4103/ijem.IJEM_89_17)

49. Nagar V, Prasad P, Gour D, Singh AR, Pal DK. Screening for diabetes among tuberculosis patients registered under revised national tuberculosis control program, Bhopal, India. J Family Med Prim Care. 2018;7(1401-5). doi:10.4103/jfmpc.jfmpc_14_18
50. Alisjahbana B, van Crevel R, Sahiratmadja E, et al. Diabetes mellitus is strongly associated with tuberculosis in Indonesia. Int $J$ Tuberc Lung Dis. 2006;10(6):696-700.

51. Gill GV, Mbanya J-C, Ramaiya KL, Tesfaye S. A Sub-Saharan African perspective of diabetes. Diabetologia. 2009;52(1):8-16. doi:10.1007/s00125-008-1167-9

Diabetes, Metabolic Syndrome and Obesity: Targets and Therapy

\section{Publish your work in this journal}

Diabetes, Metabolic Syndrome and Obesity: Targets and Therapy is an international, peer-reviewed open-access journal committed to the rapid publication of the latest laboratory and clinical findings in the fields of diabetes, metabolic syndrome and obesity research. Original research, review, case reports, hypothesis formation, expert opinion and commentaries are all considered for publication. The manuscript management system is completely online and includes a very quick and fair peer-review system, which is all easy to use. Visit http://www.dovepress.com/testimonials.php to read real quotes from published authors. 